\title{
Immunomodulatory drugs in pregnancy and lactation
}

\section{SUMMARY}

Pregnancy presents challenges for women with autoimmune diseases. It is associated with significant physiological, hormonal and immunomodulatory changes which are complex and vary according to the stage of pregnancy.

Pregnancy planning and counselling should be offered.

Autoimmune diseases such as rheumatoid arthritis tend to improve in pregnancy while systemic lupus erythematosus may increase in activity.

During pregnancy the chosen regimen should control or prevent underlying disease activity and minimise risk to the fetus. Ideally, women should be on a stable regimen before conception.

Poorly controlled disease is associated with poor outcomes for both mother and fetus, such as higher risks of pre-eclampsia, early delivery and growth restriction of the fetus.

Postpartum, there is a sudden fall in hormone concentrations, and a switch to a pro-inflammatory state. This increases the risk of relapse of many autoimmune diseases in particular rheumatoid arthritis, Crohn's disease and autoimmune hepatitis.

Many drugs are compatible with breastfeeding, but there are limited data on many of the new drugs.

\section{Introduction}

Many autoimmune diseases such as rheumatoid arthritis and systemic lupus erythematosus are more frequent in women than in men. These diseases are likely to occur during the childbearing years.

There are physiological, hormonal and immunomodulatory changes during pregnancy. Diseases with $T$ helper type 1 phenotypes (rheumatoid arthritis) may improve with pregnancy while T helper type 2 phenotypes (such as systemic lupus erythematosus) may flare in pregnancy.

Poorly controlled disease is associated with adverse pregnancy outcomes such as miscarriages, preeclampsia, growth restriction and early delivery. There are also specific maternal risks associated with the underlying disease. Pregnancy should ideally be managed by a multidisciplinary team including obstetricians, obstetric medical physicians and rheumatologists.

The Australian categorisation of drugs in pregnancy is an assessment of the risk of harm. While the categories A, B, C, D and X are a guide to the level of risk if a drug is taken during pregnancy, the system has its limitations.' For example, category D drugs may increase the incidence of fetal malformations but may still be needed to keep control of an autoimmune condition during pregnancy. Although they are both in category $\mathrm{D}$, hydroxychloroquine has been used in pregnancy while methotrexate must be avoided.

Hormone concentrations drop rapidly postpartum and there is a switch to a pro-inflammatory state.

These changes increase the risk of relapse in diseases such as rheumatoid arthritis, inflammatory bowel disease, systemic lupus erythematosus and autoimmune hepatitis.

There are potential risks to the baby from the drugs if they pass into breast milk. Often only small amounts are found so the drugs are compatible with breastfeeding. However, safety data are limited for some drugs and breastfeeding is not recommended if the mother is taking drugs such as methotrexate or mycophenolate.

\section{Pregnancy planning}

Pregnancy planning should be offered to all women of childbearing age who have an autoimmune disease. This should include education on contraception to avoid unplanned pregnancies. Pregnancy is contraindicated if the disease is poorly controlled and if the woman is taking teratogenic drugs such as methotrexate, mycophenolate or leflunomide.

Planning enables a switch to drugs that help control or prevent the activity of the disease while minimising risks to the fetus. This switch should ideally take place before conception.

\section{Kathy Paizis}

Obstetric medicine physician and Nephrologist Mercy Hospital for Women, Western Health Sunshine, Austin Health, Melbourne

\section{Keywords}

rheumatoid arthritis, systemic lupus erythematosis, teratogens

Aust Prescr 2019;42:97-101 https://doi.org/10.18773/ austprescr.2019.026 


\section{Contraception}

Contraceptive counselling is essential in women with rheumatic diseases, but is often overlooked. The choice of contraception is dependent on the severity of the disease and organ involvement, use of teratogenic drugs, underlying risk factors such as thrombotic risk, the presence of hypertension and often social circumstances.

The most effective forms of contraception are progestogen intrauterine devices (IUDs) and progestogen implants. These methods have failure rates of less than $1 \%$ per year and efficacy does not rely on adherence. There is often reluctance to use IUDs in women taking immunosuppressive drugs due to a fear of an increased risk of pelvic infections and a possible decrease in contraceptive efficacy. Data regarding the use of IUDs in immunosuppressed women are limited, but international guidelines do not consider immunosuppressive drugs to be a contraindication. ${ }^{2,3}$ IUDs are an acceptable form of contraception for both multiparous and nulliparous women. ${ }^{3}$

Progestogen implants have been associated with abnormal bleeding, but discontinuation rates are low. IUDs and progestogen implants have not been associated with an increased thrombotic risk and can safely be used in women with a history of thrombosis.

The efficacy of the combined oral contraceptive pill is user dependent with failure rates up to $9 \%$ per year as most women do not follow the strict criteria for use. A low-dose oral contraceptive pill has not been associated with increased flares in women with stable lupus. Contraindications include women more than 40 years of age, difficult to control hypertension, history of thrombosis, including conditions with increased thrombotic risk (antiphospholipid syndrome), and liver disease.

\section{Corticosteroids}

Corticosteroids are the most frequently used drugs for autoimmune diseases. They are safe in all trimesters of pregnancy (category A). Prednisolone is the preferred steroid as it is metabolised by the placenta with $10 \%$ of the maternal dose reaching the fetus.

Epidemiological studies have associated first trimester steroid use with cleft palate, ${ }^{4}$ but recent data have not confirmed this. ${ }^{5,6}$ Other reports regarding premature delivery and growth restriction have also not been reproducible. ${ }^{7}$ Steroids are associated with increased risks of hypertension, gestational diabetes, osteopenia and infections. These effects are dose dependent. Recent observational data show an incremental risk of serious infections with high-dose steroids. The incidence of infection with low-dose steroids (<10 mg) was reassuringly low. ${ }^{8}$ Screening for gestational diabetes should be performed at 16 and 28 weeks with a glucose tolerance test.

In women requiring prednisolone more than $10 \mathrm{mg}$ daily, consideration should be given to the addition of disease-modifying antirheumatic drugs (hydroxychloroquine, sulfasalazine, tumour necrosis factor inhibitors, azathioprine). This may help to improve disease control and decrease adverse pregnancy outcomes. High-dose steroids should not be withheld in women with uncontrolled disease activity. In women on long-term steroids, stress doses of intravenous steroids during labour are required. Small amounts of steroids are found in breast milk, but they are compatible with breastfeeding. In women on higher doses of steroids (more than $20 \mathrm{mg}$ prednisolone) delaying feeds for 3-4 hours after a dose can further decrease the baby's exposure to the drug.

\section{Azathioprine and mercaptopurine}

Azathioprine is commonly used in the management of transplantation, lupus nephritis, inflammatory bowel disease and autoimmune hepatitis. It is converted to mercaptopurine (6-MP) which is then enzymatically broken down to active metabolites known as thioguanines.

Despite the fact that azathioprine has not been found to be a human teratogen, it is listed as a category $D$ drug. Doses below $2 \mathrm{mg} / \mathrm{kg}$ have not been associated with an increased risk of congenital malformations (over 1300 pregnancies), stillbirth and miscarriages..$^{9,10}$ There have been links to premature delivery and intrauterine growth restriction, but this may be due to the severity of the underlying disease rather than the drug."

If azathioprine is started in pregnancy, the activity of the enzyme thiopurine S-methyltransferase (TPMT) should be measured. Decreased activity is associated with a high risk of potentially fatal bone marrow toxicity. High-dose folic acid (5 mg) is used in combination with azathioprine and mercaptopurine to prevent antifolate effects.

Azathioprine is compatible with breastfeeding as very small amounts are excreted in breast milk. The highest concentrations are found in breast milk 1-2 hours after drug ingestion. ${ }^{12}$

\section{Mycophenolate}

Mycophenolate is a very effective immunosuppressant for many autoimmune diseases in non-pregnant women. While it is classified as category D, mycophenolate is teratogenic. It should be stopped at least six weeks before conception. 
There have been consistent data supporting evidence of mycophenolate embryopathy. Typical malformations include cleft lip and palate, congenital heart defects, diaphragmatic hernia, shortened fingers, and ear, eye and renal abnormalities. ${ }^{13,14}$ Mycophenolate has also been associated with high rates of miscarriage. ${ }^{13}$

Breastfeeding is not recommended as there are limited data.

To date registry data have not shown an increased incidence of malformations in babies whose fathers took mycophenolate. Counselling these men is recommended with regards to the harm versus benefit of stopping mycophenolate when planning pregnancy with their partners. ${ }^{15}$

\section{Hydroxychloroquine}

Hydroxychloroquine is used in the management of lupus and other rheumatological conditions. It has immunomodulatory activity as well as antithrombotic, antiangiogenic and antilipidaemic actions. ${ }^{16}$ Although hydroxychloroquine is a category $D$ drug, there are now over 821 case reports of pregnancies with no increase in congenital malformations, miscarriage or stillbirth. ${ }^{9}$

Hydroxychloroquine has been associated with a decrease in flares of lupus during pregnancy and may limit the need for high-dose steroids. Observational studies have shown a decrease in the incidence of fetal heart block and cardiomyopathy in Ro antibodypositive women. In addition, hydroxychloroquine has been linked to a reduction in growth restriction and may have antithrombotic effects in women with antiphospholipid syndrome. Hydroxychloroquine should be continued in pregnancy for women with lupus and those requiring treatment for rheumatic disease. ${ }^{17}$

Hydroxychloroquine is found at low concentrations in breast milk. It is compatible with breastfeeding. ${ }^{10}$

\section{Methotrexate}

Methotrexate has many indications including rheumatological conditions - psoriatic arthritis, vasculitis and rheumatoid arthritis. It is a folic acid antagonist. $^{18}$

Despite being a teratogen, methotrexate is a category D drug. It has been associated with a high miscarriage rate (42\%) and a specific pattern of malformations and congenital heart defects (10\%). ${ }^{19}$ The timing and dosing of methotrexate may influence adverse outcomes. Recent observational data in women exposed to doses of methotrexate less than $30 \mathrm{mg}$ after conception showed a $6.6 \%$ risk of major malformations and a miscarriage rate of $20 \%{ }^{19}$ Women using less than 30 mg methotrexate per week in the three months before conception did not have an increased risk of congenital malformations or miscarriage..$^{19}$ It is currently recommended that methotrexate be stopped three months before conception. If it is taken within three months of conception, $5 \mathrm{mg}$ folic acid is recommended until at least 12 weeks of gestation, because of methotrexate's antifolate activity.

Breastfeeding is not recommended as there are insufficient data in lactation.

\section{Paternal exposure}

It is recommended that men stop methotrexate three months before conception. Two recent observational studies did not find an increased risk of congenital malformations when methotrexate (<30 mg) was used 90 days before conception..$^{20,21}$ Condoms are not required during pregnancy. Counselling men who wish to father children about the harm-benefit ratio of stopping methotrexate is advised.

\section{Sulfasalazine and mesalazine}

Sulfasalazine is often indicated for rheumatoid arthritis. Mesalazine and sulfasalazine are used in the management of inflammatory bowel disease. Sulfasalazine is category A while mesalazine is category $\mathrm{C}$. These drugs have been used in pregnancy and were not associated with an increased risk of congenital malformations, miscarriage, growth restriction or prematurity. ${ }^{10}$ There is a theoretical risk that sulfasalazine may cause folic acid deficiency as it inhibits dihydrofolate reductase and folate uptake into the cell. High-dose folic acid ( $5 \mathrm{mg}$ ) is recommended for at least one month pre-conception and up to 12 weeks after conception. ${ }^{18}$

Sulfasalazine and mesalazine are safe to use in breastfeeding. They should be used with caution if the baby is premature and has jaundice. ${ }^{10}$

\section{Paternal exposure}

Sulfasalazine and mesalazine are associated with decreased fertility in men, but not women. They decrease sperm counts and motility, but sperm counts usually return to normal two months after stopping the drug. ${ }^{22,23}$

\section{Calcineurin inhibitors}

Ciclosporin and tacrolimus have been used in the management of lupus nephritis. Tacrolimus has a more preferable adverse effect profile as it does not cause hirsutism.

Calcineurin inhibitors are not teratogenic. Although tacrolimus has been associated with a higher miscarriage rate, small for gestational age and premature delivery, this may be confounded by 
the severity of the underlying disease. ${ }^{10}$ Calcineurin inhibitors are associated with higher rates of hypertension and diabetes, so a glucose tolerance test at 16 and 28 weeks is recommended. ${ }^{24}$

Drug concentrations need to be closely monitored as they can change in pregnancy due to increased drug clearance, increased volume of distribution and less protein binding of the drug. Standard assays measure total tacrolimus concentrations and do not differentiate between unbound (free and active) versus bound drug. It is therefore recommended that concentrations are maintained at the lower end of the therapeutic range..$^{25}$

Women taking calcineurin inhibitors can breastfeed. Small amounts are found in breast milk, but no harmful effects have been reported. ${ }^{10}$

\section{Leflunomide}

Leflunomide interferes with RNA and DNA synthesis, but limited data have not shown an increased risk in congenital malformations in humans. In view of its potential risk leflunomide is considered unsafe in pregnancy (category X). ${ }^{9,10}$ Discontinuation of leflunomide before conception is insufficient for drug elimination as metabolites can be present for up to 48 months. ${ }^{26}$ Women on leflunomide before conception (48 months) or in early pregnancy should therefore undergo a colestyramine washout (8 $\mathrm{g}$ orally three times daily for 11 days) until drug concentrations are less than $0.02 \mathrm{mg} / \mathrm{L}$. Additional colestyramine is indicated if concentrations are above $0.02 \mathrm{mg} / \mathrm{L}$.

\section{Tumour necrosis factor alpha inhibitors}

Tumour necrosis factor alpha (TNF) inhibitors are commonly used in the management of inflammatory bowel disease, rheumatoid arthritis, seronegative and psoriatic arthritis. Registry data, case series and cohort analysis have not shown an increase in congenital malformations. Experience is greater with infliximab (>1200 cases), etanercept (>600 cases), adalimumab (>400) and certolizumab (>370). Data are limited on golimumab. 10,27,28 Most of the class are considered to be category $\mathrm{C}$ drugs.

The decision to continue biological therapies in pregnancy is dependent on underlying disease severity as poorly controlled disease is associated with a higher risk of adverse outcomes for mother and fetus.

The TNF inhibitors differ significantly in their molecular structure, size and half-life. Infliximab and adalimumab are IgG1 molecules, etanercept is a fusion molecule of IgG1, and certolizumab is a pegylated molecule that lacks the Fc domain. All IgG molecules are actively transported across the placenta via the Fc receptor, IgG1 being most efficiently transferred. This transfer is therefore highest for infliximab and adalimumab, low for etanercept and extremely low for certolizumab (diffusion rather than active transport) as it lacks the Fc domain. Placental transfer begins at 14 weeks and increases to delivery with maximal transfer in the last four weeks of pregnancy. ${ }^{29,30}$

To ensure no or low concentrations of the drug in cord blood it is recommended that infliximab be ceased at 18 weeks. Adalimumab should be ceased at 30-32 weeks. Etanercept and in particular certolizumab can be continued until delivery as minimal amounts are transferred to the fetus. In cases where disease activity precludes cessation of infliximab or adalimumab it is recommended that treatment be continued until delivery. ${ }^{10,28}$

There are limited, but reassuring data on breastfeeding. Small amounts are found in breast milk, but these molecules are large and absorption is thought to be minimal. ${ }^{10}$ Avoid live vaccines (rotavirus) for the first six months in newborns if a TNF inhibitor is continued in the third trimester. ${ }^{27}$ Measles-mumps-rubella vaccine is safe to be given at 12 months.

Paternal exposure to a TNF inhibitor has not been associated with adverse effects on offspring.

\section{Rituximab}

Rituximab is an antibody used in the management of antineutrophilic cytoplasmic antibody vasculitis, resistant rheumatoid arthritis and in resistant systemic lupus erythematosus. It is a category $\mathrm{C}$ drug. The timing of rituximab exposure during pregnancy has to be taken into account when assessing risk as it is an lgG1 molecule. First trimester use has been associated with an increased risk of miscarriages (potentially due to underlying disease) with no increased risk in malformations. There have been 23 reported cases of rituximab use in the second and third trimester (risk of fetal transfer) with no reports of congenital malformations or adverse fetal or maternal outcomes. However, there was a $30 \%$ incidence of cytopenias in the newborn. It is recommended that rituximab be replaced or ceased six months before conception. ${ }^{28}$

There are limited data on breastfeeding. The pharmacological properties of rituximab suggest it should be safe..$^{910,27}$ 


\section{Conclusion}

Women with underlying autoimmune disease should receive pre-pregnancy counselling. This includes advice on contraception, and often a switch to drugs that control disease and minimise harm to the fetus.
Active disease in pregnancy is associated with adverse pregnancy outcomes. Breastfeeding is often possible. Close postpartum follow-up is important as many autoimmune diseases can flare during this period. $<$

Kathy Paizis has done research funded by UCB Australia (manufacturer of certolizumab)

\section{REFERENCES}

1. Kennedy D. Classifying drugs in pregnancy. Aust Prescr 2014;37:38-40. https://doi.org/10.18773/austprescr.2014.018

2. Stringer EM, Kaseba C, Levy J, Sinkala M, Goldenberg RL, $\mathrm{Chi} \mathrm{BH}$, et al. A randomized trial of the intrauterine contraceptive device vs hormonal contraception in women who are infected with the human immunodeficiency virus. Am J Obstet Gynecol 2007:197:144.e1-8. https://doi.org/ 10.1016/j.ajog.2007.03.031

3. Curtis KM, Tepper NK, Jatlaoui TC, Berry-Bibee E, Horton LG, Zapata LB, et al. US medical eligibility criteria for contraceptive use, 2016. MMWR Recomm Rep 2016;65:1-104. http://dx.doi.org/10.15585/mmwr.mm6637a6

4. Park-Wyllie L, Mazzotta P, Pastuszak A, Moretti ME, Beique L, Hunnisett $L$, et al. Birth defects after materna exposure to corticosteroids: prospective cohort study and meta-analysis of epidemiological studies. Teratology 2000;62:385-92. https://doi.org/10.1002/ 1096-9926(200012)62:6<385::AID-TERA5>3.0.CO;2-Z

5. Hviid A, Mølgaard-Nielsen D. Corticosteroid use during pregnancy and risk of orofacial clefts. CMAJ 2011;183:796-804 https://doi.org/10.1503/cmaj.101063

6. Bay Bjørn AM, Ehrenstein V, Hundborg HH, Nohr EA Sørensen HT, Nørgaard M. Use of corticosteroids in early pregnancy is not associated with risk of oral clefts and other congenital malformations in offspring. Am J Ther 2014;21:73-80. https://doi.org/10.1097/MJT.0b013e3182491e02

7. Al Arfaj AS, Khalil N. Pregnancy outcome in 396 pregnancies in patients with SLE in Saudi Arabia. Lupus 2010;19:1665-73. https://doi.org/10.1177/0961203310378669

8. Desai RJ, Bateman BT, Huybrechts KF, Patorno E, Hernandez-Diaz S, Park Y, et al. Risk of serious infections associated with use of immunosuppressive agents in pregnant women with autoimmune inflammatory conditions: cohort study. BMJ 2017;356:j895. https://doi.org/10.1136/bmj.j895

9. Flint J, Panchal S, Hurrell A, van de Venne M, Gayed M, Schreiber K, et al.; BSR and BHPR Standards, Guidelines and Audit Working Group. BSR and BHPR guideline on prescribing drugs in pregnancy and breastfeeding-Part I: standard and biologic disease modifying anti-rheumatic drugs and corticosteroids. Rheumatology (Oxford) 2016;55:1693-7. https://doi.org/10.1093/rheumatology/kev404

10. Götestam Skorpen C, Hoeltzenbein M, Tincani A, Fischer-Betz R, Elefant E, Chambers $C$, et al. The EULAR points to consider for use of antirheumatic drugs before pregnancy, and during pregnancy and lactation. Ann Rheum Dis 2016;75:795-810. https://doi.org/10.1136/annrheumdis-2015-208840

11. Cleary BJ, Källén B. Early pregnancy azathioprine use and pregnancy outcomes. Birth Defects Res A Clin Mol Teratol 2009;85:647-54. https://doi.org/10.1002/bdra.20583

12. Sau A, Clarke S, Bass J, Kaiser A, Marinaki A, Nelson-Piercy C. Azathioprine and breastfeeding: is it safe? BJOG 2007;114:498-501. https://doi.org/10.1111/j.1471-0528.2006.01232.x

13. Sifontis NM, Coscia LA, Constantinescu S, Lavelanet AF, Moritz MJ, Armenti VT. Pregnancy outcomes in solid organ transplant recipients with exposure to mycophenolate mofetil or sirolimus. Transplantation 2006;82:1698-702 https://doi.org/10.1097/01.tp.0000252683.74584.29

14. Hoeltzenbein M, Elefant E, Vial T, Finkel-Pekarsky V, Stephens S, Clementi M, et al. Teratogenicity of mycophenolate confirmed in a prospective study of the European Network of Teratology Information Services. Am J Med Genet A 2012;158A:588-96. https://doi.org/10.1002/ajmg.a.35223

15. Lopez-Lopez I, Rodelo-Haad C, Agüera ML

Cabello-Jabalquinto R, Esquivias-Motta E, Navarro MD, et al. Administration of mycophenolic acid is not associated with malformations in descendants from kidney transplanted males. PLoS One 2018:13:e0202589. https://doi.org/10.1371/ journal.pone.0202589
16. Wallace DJ, Gudsoorkar VS, Weisman MH, Venuturupalli SR New insights into mechanisms of therapeutic effects of antimalarial agents in SLE. Nat Rev Rheumatol 2012;8:522-33. https://doi.org/10.1038/nrrheum.2012.106

17. Knight CL, Nelson-Piercy C. Management of systemic lupus erythematosus during pregnancy: challenges and solutions. Open Access Rheumatol 2017:9:37-53. https://doi.org/ 10.2147/OARRR.S87828

18. Hernández-Díaz S, Werler MM, Walker AM, Mitchell AA. Folic acid antagonists during pregnancy and the risk of birth defects. N Engl J Med 2000;343:1608-14. https://doi.org/ 10.1056/NEJM200011303432204

19. Weber-Schoendorfer C. Chambers C, Wacker E, Beghin D, Bernard N, Shechtman S, et la. Pregnancy outcome after methotrexate treatment for rheumatic disease prior to or during early pregnancy: a prospective multicenter cohort study. Arthritis Rheumatol 2014:66:1101-10. https://doi.org/ 10.1002/art.38368

20. Weber-Schoendorfer C, Hoeltzenbein M, Wacker E Meister R, Schaefer C. No evidence for an increased risk of adverse pregnancy outcome after paternal lowdose methotrexate: an observational cohort study. Rheumatology (Oxford) 2014:53:757-63. https://doi.org/ 10.1093/rheumatology/ket390

21. Eck LK, Jensen TB, Mastrogiannis D, Torp-Pedersen A Askaa B, Nielsen TK, et al. Risk of adverse pregnancy outcome after paternal exposure to methotrexate within 90 days before pregnancy. Obstet Gynecol 2017;129:707-14. https://doi.org/10.1097/AOG.0000000000001936

22. O'Moráin C, Smethurst P, Doré CJ, Levi AJ. Reversible male infertility due to sulphasalazine: studies in man and rat. Gut 1984;25:1078-84. https://doi.org/10.1136/gut.25.10.1078

23. Shin T, Kobori Y, Suzuki K, Iwahata T, Yagi H, Soh S, et al. Inflammatory bowel disease in subfertile men and the effect of mesalazine on fertility. Syst Biol Reprod Med 2014;60:373-6. https://doi.org/10.3109/19396368.2014.952391

24. Chakkera HA, Kudva Y, Kaplan B. Calcineurin inhibitors: pharmacologic mechanisms impacting both insulin resistance and insulin secretion leading to glucose dysregulation and diabetes mellitus. Clin Pharmacol Ther 2017;101:114-20. https://doi.org/10.1002/cpt.546

25. Zheng S, Easterling TR, Umans JG, Miodovnik M, Calamia JC Thummel KE, et al. Pharmacokinetics of tacrolimus during pregnancy. Ther Drug Monit 2012;34:660-70. https://doi.org/ 10.1097/FTD.0b013e3182708edf

26. Østensen M, Khamashta M, Lockshin M, Parke A, Brucato A Carp H, et al. Anti-inflammatory and immunosuppressive drugs and reproduction. Arthritis Res Ther 2006;8:209. https://doi.org/10.1186/ar1957

27. Alijotas-Reig J, Esteve-Valverde E, Ferrer-Oliveras $\mathrm{R}$ Llurba E, Gris JM. Tumour necrosis factor-alpha and pregnancy: focus on biologics. An updated and comprehensive review. Clin Rev Allergy Immunol 2017;53:40-53. https://doi.org/10.1007/s12016-016-8596-x

28. Gerosa M, Argolini LM, Artusi C, Chighizola CB. The use of biologics and small molecules in pregnant patients with rheumatic diseases. Expert Rev Clin Pharmacol 2018;11:987-98. https://doi.org/10.1080/17512433.2018.1525293

29. Kane SV, Acquah LA. Placental transport of immunoglobulins: a clinical review for gastroenterologists who prescribe therapeutic monoclonal antibodies to women during conception and pregnancy. Am J Gastroenterol 2009;104:228-33. https://doi.org/10.1038/ajg.2008.71

30. Malek A, Sager R, Kuhn P Nicolaides KH, Schneider H. Evolution of maternofetal transport of immunoglobulins during human pregnancy. Am J Reprod Immunol 1996;36:248-55. https://doi.org/10.1111/j.1600-0897.1996.tb00172.x

\section{SELF-TEST} QUESTIONS

True or false?

3. A weekly dose of methotrexate to control rheumatoid arthritis can continue until the third trimester of pregnancy. 4. Calcineurin inhibitors, such as ciclosporin, should be stopped at least three months before a planned pregnancy.

Answers on page 113 